\title{
LAS UNIDADES DISTRIBUCIONALES EN EL ANÁLISIS DEL RELATO
}

En 1966 Barthes se basó en Tomachevski, en Propp, en Hjelmslev y en Benveniste, principalmente, al redactar su "Introducción al análisis estructural del relato", uña de las más útiles aportaciones de los teóricos de esa época. En este trabajo ofreció por primera vez un amplio esquema globalizador de todos los problemas y todas las etapas de estudio de este tipo de textos. Además, en él también sienta las bases para la segmentación del discurso en unidades de significación a las que llama "funciones".

En efecto, antes que él, Tomachevski (Temática, 1925) había llamado "motivos" a tales unidades del relato, de las que dijo que son las partículas más pequeñas del "tema" (la historia) que, combinadas entre sí, "constituyen el armazón temático de la obra", y que residen en unidades que gramaticalmente son proposiciones (como después lo serán para Propp, Barthes, "Todorov, Hendricks, etcétera): "Ha caído la tarde", "Raskolnikov asesinó a la vieja", son sus ejemplos.

Además, con la perspicacia y longitud de visión características en los miembros del movimiento formalista ruso, Tomachevski fue aún más lejos y clasificó los motivos en "asociados", que son "los que no pueden ser excluidos" y "cuentan sobre todo en la "trama" (equivalente al orden canónico de los elementos en la historia) $y$, por otra parte, los motivos "libres", "Ios que pueden extirparse sin lesionar la sucesión cronológica y causal de los acontecimientos". Estos últimos son las "catálisis" de Barthes.

Tomachevski atribuye a estos motivos libres un mayor papel en el "argumento" (construcción regida por el orden artístico y no por el canónico) pero también, al llamarlos más adelante "motivos marginales" y "detalles", parece establecer para ellos una jerarquía inferior a la de los motivos 
asociados, criterio que parece similar al que después adoptará Barthes.

Hasta aquí, el punto de vista de Tomacherski para describir los motivos consiste en considerar el modo como se relacionan entre sí; pero además los clasifica en motivos "dinámicos", si modifican la situación, y motivos "estáticos" si no la modifican; basándose al hacerlo ya no en su tipo de relación sino en la función que desempeñan en el desarrollo de la historia narrada. También estas nociones subyacen respectivamente en las de "nudos" y "catálisis" de Barthes.

De Propp (Morfología del cuento, 1928) procede la noción de función en tanto que unidad morfológica mínima que se describe como "acción de un personaje, definida por su situación en el curso del relato" y por su "significación en el desarrollo de la intriga". De Hjelmslev (Prolegómenos a una teoria del lenguaje, 1943) proviene la descripción del tipo de relación que guardan entre sí las unidades distribucionales: interdependencia o solidaridad o doble implicación, de naturaleza recíproca, entre los nudos; implicacación simple entre nudos y catálisis. En fin, se debe a Benveniste ("Los niveles del análisis lingüístico", 1962) el procedimiento de identificación de las unidades del texto (es decir, de los "elementos" de su "lenguaje"); identificación previa y necesaria al análisis, puesto que éste consiste para él en delimitar esos elementos "a través de las relaciones que los unen". Según Benveniste, el análisis se sustenta en "dos operaciones que se gobiernan una a otra y de las que dependen todas las demás: la segmentación y la sustitución".

Benveniste, en efecto, recomienda que el texto, independientemente de sus dimensiones, sea primeramente segmentado en porciones hasta llegar a "elementos no descomponibles", y agrega: "paralelamente se identifican tales elementos por las sustituciones que admiten". Hasta aquí, Benveniste está refiriéndose a fonemas y palabras. Su objeto es llegar a la descripción del "método de distribución" que "consiste en definir cada elemento por el conjunto de los alrededores en que se presenta", por medio de la doble relación: sintagmática ("con los demás elementos simultáneamente presentes en la misma porción del enunciado") y 
paradigmática ("con los demás elementos mutuamente sustituibles").

Lúcida y oportunamente Barthes advirtió en todas las anteriores nociones los rasgos que las hacen complementarias y que permiten su combinación tal como él la propone al establecer como unidades distribucionales aquellas que admiten relaciones dentro del mismo nivel, y como unidades integradoras aquellas que admiten relaciones con unidades de un nivel diferente. En ambos casos, los elementos de nivel inferior a la unidad no son otras unidades sino simplemente "segmentos formales" de la misma.

Sobre tales bases propone, pues, Barthes, en su "Introducción. . ", segmentar el relato en "unidades funcionales" que son "unidades de contenido", a las que describe como "narrativas" y como "mínimas" y también como "segmentos de discurso" (a los que antes Propp y en esa misma época Bremond denominan "funciones"). Barthes clasifica esas unidades, primeramente, en dos grandes grupos: unidades distribucionales y unidades integradoras (a las que se aludió en el párrafo anterior). Luego, dentro de las primeras considera los "nudos", que "no pueden ser excluidos" (como los motivos asociados de Tomachevski) y que Barthes caracteriza como constituyentes de un "hilo narrativo", como "resortes de una actividad" y "detonadores del desarrollo progresivo de una acción" cuyo sentido se capta en. las unidades subsecuentes con las que mantienen una relación sintagmática de carácter distribucional y dentro del mismo nivel, y cuya supresión no es posible porque perturbaría la sucesión lógico-temporal de las acciones cuyo conjunto forma el armazón o esqueleto del relato.

Dentro de las unidades integradoras menciona los "índices" o "indicios" y las "informaciones". Los primeros procuran los rasgos que permiten caracterizar a los personajes en el nivel de las funciones e.investirlos de las categorías actanciales en el nivel de las acciones. Las segundas ofrecen Jos datos que sirven "para identificar y situar los objetos $y$ los seres en el tiempo y en el espacio" que funge como escenario de la historia, y su sentido no se capta "después" sino "más arriba", en el nivel de las acciones o en el nivel 
del discurso que es donde se relacionan con sus correlatos durante la lectura, integrando así su significado al sentido total del relato.

Barthes adjudica conjuntamente a las catálisis, los índices y las informaciones un carácter "expansivo" que consiste en su capacidad de proliferar en número infinito dentro de un espacio localizable entre los nudos. Estas expansiones parecen corresponder a un desarrollo de los motivos estáticos de Tomachevski, pues tanto las catálisis como las informaciones y los índices poseen una naturaleza "infinitamente catalizable".

Pero en esta teoría hay un punto neurálgico que se advierte más fácilmente cuando se trata de llevarla a la práctica, pues parece haber una contradicción donde quizá sólo falta desarrollo y un mayor número de ejemplos. En autores que posteriormente se han ocupado del problema no hay una puntualización suficientemente clara y explícita que sin embargo es necesaria. Bobes Naves esclarece otras cuestiones pero cuando se refiere a ésta es bastante vaga: "el relato discurre hacia su desenlace a través de las funciones encadenadas o se retrasa y remansa por medio de acciones virtuales o mediante la reiteración de alguna función". Hendricks también trata de aclarar el problema de la funcionalidad de las catálisis en sus aplicaciones a ejemplos, y así cuando está identificando la cadena de acciones ofrece una explicación confusa: "esto es una acción ("el negro abrió las persianas") pero su única misión es posibilitar la observación de un detalle descriptivo". $\mathrm{Y}$ en otro ejemplo ("se elevó perezosamente... polvo oscuro") dice: "se trata de un nuevo enunciado de acción, pero instintivamente puede parecer descriptivo. (Se puede ver que el sujeto del verbo es inanimado)". Este mismo autor más tarde, cuando habla del "resumen sintagmático que produce una representación de la estructura del argumento" dice que "un paso preliminar es la escisión de todos los enunciados de acción no funcionales" (en el sentido de, Propp: elementos que componen la escena, la resumen, la anticipan, etcétera), es decir, los enunciados omisibles. Segre, por otra parte, que dedica muchas páginas a detectar $y$ proponer soluciones para 
este tipo de puntos débiles de la teoría del relato, elude en este caso la aclaración y se limita a citar literalmente a Barthes.

El problema consiste en que el estatuto de las catálisis tal como lo presenta Barthes no es claro: $\mathrm{Y}$ es importante aclararlo porque esta noción, aplicada al análisis de las funciones, se relaciona estrechamente con lo que Todorov llama "modos de lo real" y "modos de la hipótesis" que facilitan la distinción entre historia y discurso, y conducen más tarde al análisis de secuencias; pero además, esta noción arroja luz también sobre el problema de la caracterización de los personajes, el de los desfases de temporalidad que se producen entre historia y discurso, que afectan al orden, la duración, o la frecuencia, y sobre la noción de niveles de la diégesis, tal como los trata Genette.

La naturaleza de las catálisis es distribucional pero su relación, descrita como paradigmática, es a veces, aun en ejemplos del mismo Barthes, consecutivo-causal. Para este autor, dicha relación es de implicación simple respecto a los nudos, y libre respecto a las unidades integradoras con las cuales comparte su calidad de "expansión", y agrega que constituyen tanto los "incidentes menudos" como las "detalladas descripciones", y que son "notaciones subsidiarias" de naturaleza complementaria y de "funcionalidad atenuada, unilateral y parásita". Como se ve, el problema tiene más de una faceta: por una parte, la jerarquía que denotan calificativos tales como "subsidiaria", "complementaria", "atenuada", "parásita", no tiene fundamento, pues a pesar de que las catálisis dependen de los nudos, pueden aparecer en un número infinitamente mayor que éstos, y su papel puede ser más importante, si no para la existencia de la historia relatada, sí para su sentido total.

Esto ha sido advertido por los analistas. Todorov reconoce, por ejemplo, que "las catálisis a veces pueden ocupar la mayor parte de la obra", y probablemente un estudio diacrónico comprobaría que esto tiende a ocurrir en mayor medida en la literatura moderna. Los miembros del Grupo $\mu$ también mencionan - como desviación- este hecho, e interpretando a Barthes del mejor modo posible hablan de 
"nudos descriptivos" y "nudos narrativos". Ellos mencionan las catálisis como "extensiones descriptivas que tienen lugar en el desarrollo de las funciones cardinales" y que no pueden ser disociadas de los nudos "porque forman cuerpo con ellos". Esto último ya no resulta muy claro pues, por el contrario, se diferencian formal y funcionalmente lo suficiente para que puedan distinguirse, alternar y contrastar.

A partir de todos estos antecedentes, y buscando, además, ciertos acercamientos que parecen útiles para la teoría del relato tal como la plantean los mencionados Todorov, Genette y Grupo $\mu$, y tal como Weinrich explica el funcionamiento de la temporalidad de los verbos, trataré de aclarar un poco más el carácter, el alcance y la funcionalidad de las catálisis. La correlación entre los desarrollos de los diversos autores permite que procedimientos parciales y pequeños pasos de análisis resulten complementarios $y$ ofrezcan un resultado global. Consideremos las siguientes observaciones:

1. Las unidades distribucionales que resultan de la segmentación del texto son proposiciones que comprenden verbos cuya naturaleza varía según la unidad sea un nudo narrativo, o bien un nudo descriptivo de naturaleza catalítica.

2. Los nudos narrativos se construyen solamente con verbos de acción cuya sucesión constituye el "alma" del eje sintagmático en la historia. La lista de estos verbos de acción constituye la parte principal de la historia, tanto en la fábula como en la intriga.

2.1. Para que formen parte de un nudo narrativo las formas de los tiempos de acción, deben corresponder a lo que Todorov llama "modos de lo real", que "se perciben como designando acciones que verdaderamente han tenido lugar".

2.2. Las formas de los verbos de acción en los nudos naxrativos pueden pertenecer tanto a lo que Weinrich llama "grupo temporal I" (flexiones del presente) que dominan en la "situación comunicativa de los diálogos", en la que no se narra sino se comenta, como al "grupo temporal II" (flexiones del pretérito perfecto simple y del pretérito imperfecto) que dominan en la situación comunicativa narrativa; es decir, tanto las flexiones que corresponden a las ac- 
ciones narradas como las que tocan a las acciones representadas.

Los nudos descriptivos se construyen de dos maneras:

- con verbos que expresan cualidades o maneras de ser o de estar habitualmente;

- con los mismos verbos que participan en la construcción de los nudos narrativos, es decir, con verbos de acción pero en circunstancias que comprimen, distienden, anticipan, evocan, repiten o trasladan la dimensión temporal de una diégesis a otra.

Las formas de los verbos de acción de los nudos descriptivos corresponden a lo que Todorov llama "modos de la hipótesis" o de "lo irreal" porque expresan acontecimientos futuros o posibles o pertenecientes a otra historia, a una metadiégesis; o bien son objetos directos de otros verbos, o están en oraciones subordinadas finales.

Pero el problema está en que las formas de los verbos de acción de los nudos descriptivos pueden ser también las de "modos de lo real", y en ese caso corresponden a una puntualización extremadamente detallada de las acciones que, por ello mismo, son resumibles en macroproposiciones para los fines de la elaboración del resumen requerido para analizar las funciones. Por ejemplo: todas las acciones menudas que constituyen los actos de fumar, arrellanarse en el asiento, hojear revistas, observar en varios turnos y en diversas direcciones el entorno, pueden entrar al resumen de las funciones en una sóla macroproposición: "X esperó largo rato", siempre que las acciones anteriores y las subsecuentes sean del orden de: "se trasladó a la estación", "alcanzó a transbordar en París", "tuvo una entrevista con su socio", etcétera, puesto que tanto el ritmo establecido como sus rupturas son relativos. Muchas de estas acciones pueden ser consideradas individualmente en un relato del tipo del Ulises de Joyce.

De aquí se infiere la importancia del manejo de una noción clara de catálisis, ya que si nos proponemos un estudio completo de la totalidad de una obra, a partir del análisis de funciones podremos: 
-elaborar un buen resumen, necesario para estudiar, sobre él, las secuencias y para establecer la fábula y distinguirla de la intriga;

-identificar a los protagonistas caracterizados a través de sus propias acciones y a aquellos que lo son a través de descripciones del narrador o del narrador-personaje;

-identificar, describir y semantizar los desfases de temporalidad que afectan a la duración, los que alteran el orden y los que se producen por modificaciones de la frecuencia;

- -establecer los grados de la narración o niveles de la diégesis (y con ello ubicar con facilidad al narrador).

$\mathrm{Si}$ al realizar cada una de estas operaciones tenemos presentes los criterios de Barthes, Todorov, Grupo $\mu$ y Genette, sobre todo, hallamos que recíprocamente se complementan y se aclaran facilitando la comprensión del texto. Ahora bien, al combinar la teoría y los procedimientos, hay que recordar que Genette no hace análisis de funciones ni resumen.

Combinando en la práctica todas las nociones hasta aquí mencionadas hallaremos que:

I. Toda descripción tiene una función catalítica y constituye una pausa, anisocronía o desfase temporal que afecta a la duración de la enunciación si se considera con respecto a la extensión del texto que contiene lo enunciado. Se trata de una catálisis expansiva que se produce por la proliferación - como le llama Barthes- de nudos descriptivos que pueden estar a cargo de verbos de acción ya sea en los modos "de lo real" o "de la hipótesis", pero también a cargo de verbos que signifiquen cualidad o estado.

Cuando los verbos son de acción y están en los modos "de lo real", la pausa consiste en un relajamiento del ritmo de la historia, y no en una suspensión de la misma; es una desaceleración. En tal caso, para los fines de la elaboración del resumen (necesario para el análisis de funciones) dichos verbos quedan incluidos en una sola macroproposición. En este tipo de catálisis cada acción compleja queda desmenu- 
zada en sus detalles que son todas las acciones menudas comprendidas en ella; lo que significa que se expande el tiempo del discurso a expensas del tiempo de la historia, pues ésta ocurre de todos modos en el mismo lapso, ya sea que el narrador detalle las pequeñas acciones que la constituyen o que no lo haga. El acto de lanzar un cubo de agua arrojando su contenido a cierta distancia (como lo hace uno de los personajes de un cuento de Revueltas) puede ser descrito con un número imprescindible de palabras o bien desmenuzando dicho gesto en una infinidad de pequeños movimientos eslabonados, como en cámara lenta, que en el análisis quedan comprendidos en uno, mediante el empleo de una sola macroproposición capaz de expresar todo el movimiento. El primer procedimiento puede constituir una catálisis reductiva si la narración está construida a un ritmo que corresponde al del segundo procedimiento; y si es al contrario, la función catalítica no es reductiva sino expansiva.

Guando se trata de verbos de acción en los modos "de la hipótesis", o de verbos que significan cualidad o estado, la pausa no es ya una simple desaceleración sino una completa suspensión de la historia (así la describe Genette) mientras sigue desarrollándose el proceso discursivo, como cuando se describe un ambiente o un personaje. En este caso, la catálisis resulta una unidad de carácter integrador más que distribucional, de donde se desprende que son unidades híbridas.

En la pausa, pues, varía el grado de desajuste temporal (desaceleración, cuando la acción sigue transcurriendo pausadamente, o suspensión cuando deja de transcurrir) según sea la naturaleza de los verbos.

2. Todo resumen constituye un tipo de anisocronía opuesto a la pausa por lo que también afecta a la duración. Se trata de una catálisis reductiva. Un relato isócrono, en cambio, presenta una velocidad que convencionalmente consideramos constante e igual en ambas temporalidades, lo que se supone que ocurre en el diálogo o, como lo llama Genette, la escena. Por otra parte, la elipsis o supresión de 
nudos de significación inferible a partir de los nudos precedentes y subsecuentes, es un fenómeno de naturaleza catalítica porque altera el ritmo de desarrollo de los hechos relatados y constituye un desface de temporalidades, porque se omite el discurso pero se deduce la historia.

3. En cuanto a las anacronías, las analepsis o retrospecciones introducen en el "aquí-ahora" de la escena imaginaria donde se supone que ocurre la acción, un "aquí-ahora" que no es presente sino que fue presente. Por su parte, las prolepsis o anticipaciones introducen un "aquí-ahora" que será o podria llegar a ser presente. Ambas constituyen anacronias y el desfase que producen altera el paso del relato, pero no porque afecten a la relación entre duración de la historia y longitud del texto que la expresa, sino porque la desaceleración se debe aquí a interrupciones introducidas por el establecimiento de un orden artificial que es el de la intriga. Si la narración de la vida adulta de un personaje se interrumpe constantemente con intercalaciones que son pasajes de su infancia, aunque la posición del narrador nos permita considerarlos como fragmentos de la misma diégesis sin que su dimensión espacio-temporal sea idéntica, las interrupciones alteran formalmente la enunciación pues el mantenimiento de la coherencia en un orden que no es lógico-temporal requiere el empleo de un mayor número de elementos enunciadores tales como anafóricos, deícticos, etcétera; y naturaimente altera también la temporalidad de la lectura. Las anacronías son asímismo, pues, de naturaleza catalítica. Muchas de ellas son relatos metadiegéticos generados a partir de la narración en primer grado a guisa de digresiones que la interrumpen y se introducen en ella subordinándosele pero con su propio narrador, su propia plataforma espacio-temporal y su propio ritmo. En no pocos casos, además, el manejo de todos estos recursos en la metadiégesis funciona como elemento indicial que permite caracterizar al narrador personaje, por lo que también en este caso las catálisis se comportan como unidades integradoras, luego híbridas. Por ejemplo, el guardagujas del cuento de Arreola, que dialoga con el personaje mientras llega el tren, es 
caracterizable a partir de sus parlamentos que constituyen una variada serie de catálisis.

Las anacronías suelen ser al mismo tiempo anisocronías, - sea, catálisis expansivas (pausas) o reductivas (resúmenes); de todos modos su efecto sobre la diégesis, por su carácter de agregados a ella, es siempre expansivo.

4. La repetición de un mismo nudo o la expresión de la ocurrencia de una misma acción muchas veces, mediante el empleo de la forma verbal de aspecto iterativo, produce un tipo de desfase temporal que afecta a la frecuencia al evitar que coincidan $\mathrm{x}$ número de ocurrencias discursivas con $\mathrm{x}$ número de ocurrencias de los hechos expresados por ellas. El relato llamado por Genette "repetitivo" narra varias veces lo ocurrido una sola vez y así prolonga, de otra manera, la duración del discurso. El relato "iterativo", en cambio, que implica condensación y narra una sola vez lo ocurrido varias veces, constituye una catálisis reductiva que mediante dicho procedimiento acorta la duración (medida en términos de extensión) del discurso.

Para tener presente durante el análisis una idea clara acerca de cómo identificar los verbos de acción que constituyen nudos descriptivos porque fragmentan en acciones menudas y simples otras complejas y, asimismo, cómo reconocer los verbos de acción en los modos "de la hipótesis", que constituyen acciones puramente discursivas porque se presentan como pensadas por el narrador o los personajes (abstracciones, generalizaciones, reflexiones, hipótesis, citas), o como hechos que ocurren en una metadiégesis; o bien que sean objetos directos de otros verbos o estén en oraciones subordinadas finales, o manifiesten hechos futuros (prolepsis) o pasados (analepsis) o posibles, o que caractericen o describan modos de ser o de ejecutar otras acciones mediante oraciones subordinadas al núcleo del sujeto o al del predicado; para tener todo esto presente, repito, es de la mayor utilidad relacionarlo con los niveles de la diégesis, las anisocronías y los desfasamientos de frecuencia y con el concepto de catálisis que proviene del análisis de funciones de Barthes ya que de esta manera se enriquece y se aclara; 
de esa manera podríamos pensar en un resumen conclusivo como el siguiente:

\section{Catálisis I}

\begin{tabular}{|c|c|c|c|}
\hline $\begin{array}{l}\text { naturaleza de } \\
\text { las catálisis }\end{array}$ & & $\begin{array}{l}\text { la tempo- } \\
\text { olucrados }\end{array}$ & $\begin{array}{l}\text { fenómenos } \\
\text { catalíticos }\end{array}$ \\
\hline reductivas & $\begin{array}{l}\text { duración } \\
\text { frecuencia } \\
\text { duración } \\
\text { orden } \\
\text { frecuencia }\end{array}$ & pausa & $\begin{array}{l}\text { resumen } \\
\text { elipsis } \\
\text { relato iterativo } \\
\left\{\begin{array}{l}\text { suspensión } \\
\text { desáccleración }\end{array}\right. \\
\left\{\begin{array}{l}\text { analepsis } \\
\text { prolepsis }\end{array}\right. \\
\text { relato repetitivo }\end{array}$ \\
\hline
\end{tabular}

Catálisis II

Tipos de verbos con que se construyen las catálisis

a) Verbos que significan cualidad o estado.

b) Verbos de acción en los modos de la hipótesis (acciones discursivas)

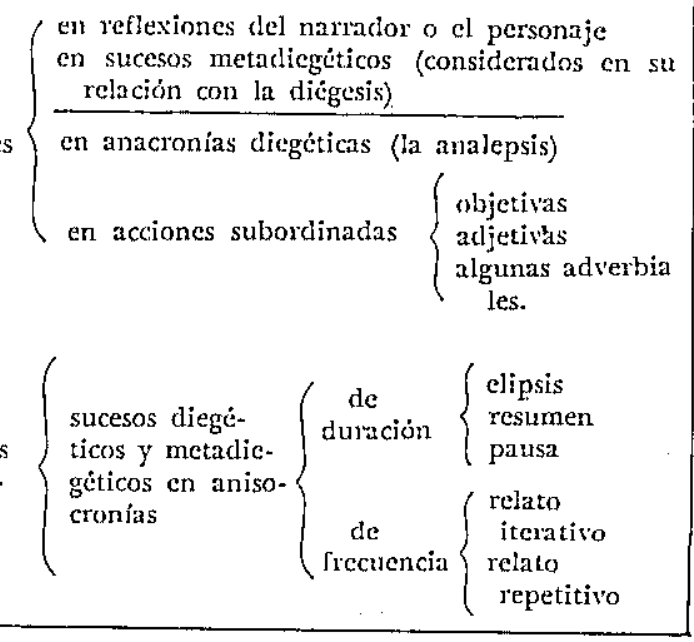

c) Verbos de acción en los modos de lo real: acciones menudas resumibles en macroestructuras y aspecto iterativo de las acciones

El manejo de las catálisis puede producir una serie de efectos como son los relacionados con:

a) las variaciones del ritmo interno del relato, de naturaleza anisocrónica, logradas mediante la alteración tanto de 
la duración como de la frecuencia y el orden de ambas temporalidades, la del discurso y la de la historia. Aparte de que evitan la monotonía, pueden semantizarse de manera que varía en cada caso;

b) las variaciones de la tensión del relato que pueden 1ograrse:

$\rightarrow$ mediante anisocronías, posponiendo la acción para dar lugar a la expansión del discurso, o bien desacelerándola paralelamente a la expansión del discurso, en la pausa;

-mediante anacronías, introduciendo un orden diferente al canónico, con lo que se anticipan o posponen las acciones más significativas para la diégesis, de manera que se agudicen las crisis y se intensifique el suspenso;

c) el énfasis, que paradójicamente puede obtenerse tanto de las catálisis expansivas mediante la dedicación de $\mathrm{x}$ número de ocurrencias a la narración de una ocurrencia de la historia, como de las catálisis reductivas seleccionando sólo las acciones más importantes en el resumen, o eludiéndolas mediante la elipsis.

De todo lo anterior se desprende que las catálisis son unidades funcionales de naturaleza hibrida - distribucional e jntegradora- que pueden tener o no verbos de acción en los modos de lo real. Que son nudos descriptivos que pueden estar constituidos por acciones narrativas o por acciones discursivas. Además, que toda metadiégesis es de naturaleza catalítica por ser un relato interno, secundario, que crece dentro de la diégesis y a expensas de su temporalidad.

El ritmo resultante de las catálisis anisocrónicas podría diagramarse sobre una especie de tetragxama doble (uno para la diégesis y otro para la metadiégesis) del modo siguiente para el inicio del cuento "El guardagujas" de Juan José Arreola: 
+ (pausa o exten.

sión discursiva)

\begin{tabular}{lll}
\hline & (escena o diálogo) & \\
\hline$=$ (resumen o reducción) & & \\
\hline 0 & (supresión o elipsis) & \\
\hline & & \\
\hline (pausa) & & \\
\hline$=$ (escena) & \\
\hline- (resumen) & \\
\hline O (elipsis) & & \\
\hline
\end{tabular}

* Cada acción corre a cargo de uno de los personajes: 0 o X.

En este relato los nudos de la narración primaria son muy pocos pues podrían inclusive reducirse a sólo tres macropro: posiciones: "E1 forastero llegó", "Esperó el tren conversando con un ferocarrilero", "El tren Ilegó a la estación". El resto de los nudos constituyen catálisis diegéticas o bien metadiegéticas, cuyo relato está a cargo de uno de los personajes de la diégesis. Dichas catálisis son de distintos tipos:

a) pausas dilatorias en las que, por ejemplo, se desmenuzan y detallan los gestos que manifiestan la llegada o la espera;

b) pausas elípticas o suspensiones en las que se suspende Ia diégesis para dar paso a la descripción de un modo habitual de ser, por ejemplo el del funcionamiento del sistema de los ferrocarriles;

c) anacronías metadiegéticas de muy diversas extensiones, relatadas por uno de los personajes, del tipo de: "Yo he visto pasar muchos trenes en mi vida y conocí a algunos viajeros que pudieron abordarlos", o como aquella otra, más extensa, en que se narra la fundación por accidente de una nueva aldea;

d) nudos descriptivos expresados con verbos de acción en 
los modos de la hipótesis y generalmente combinados con un tipo de anacronía: la prolepsis o anticipación. Es un ejemplo la de la próxima construcción de un nuevo tramo de los ferrocarriles, o bien la de las tentaciones y los espejismos que sufrirá el viajero en su próximo viaje. En ambos casos los verbos expresan acciones puramente discursivas. Estos dos últimos tipos de catálisis son también suspensiones de la cadena de hechos que constituye la narración primaria, la que en este caso es el punto de apoyo a partir del cual se generan unas metadiégesis pretéritas y otras hipotéticas o futuras, de tal modo que la diégesis se abandona momentáneamente para narrar otras historias que pueden ser:

-ya ocurridas

- posibles

- planeadas como futuras

-recomendadas (que también se cumplirían como ocurencias futuras: "alquile usted un cuarto...")

o bien para describir:

- los modos de ser de las personas y los objetos ("Hace circular los trenes en lugares intransitables")

-o los modos de hacer, es decir, de realizar las acciones: ("llegó sin aliento... le había fatigado... se enjugó el rostro... miró los rieles... consultó su reloj")

De las catálisis anacrónicas resultan como efectos tanto la metadiégesis como la intriga. En efecto, tanto la prolepsis como la analepsis son metadiegéticas si su narrador es intradiegético y si su plataforma espacio-temporal es otra distinta a la de la diégesis. En cambio, si el narrador es extradiegético o bien intradiegético y autodiegético simultáneamente, las anacronías son intradiegéticas y constituyen la intriga $u$ ordenamiento artificial o artístico de la historia, opuesta a la fábula u ordenamiento natural o canónico de la misma. El diagrama de las anacronías nos da precisamente el grado de desfasamiento entre la temporalidad de la fábula y la de la intriga. Si la fábula estuviera constituida 
por la siguiente lista de nudos, indicados por letras y si el orden en que el narrador da cuenta de los mismos estuviera señalado por los números, el diagrama de las catálisis añcrónicas sería:

llegó se introdujo observó intervino huyó fue perseguido

(a)

(b)

(c)

(d)

(c)

(2)

(4)

(5)

La sucesión de las letras marca la fábula; la de los números, la intriga, y las discrepancias entre ambos dan cuenta del desfasamiento entre las dos temporalidades, lo que también puede observarse estableciendo la correspondencia que propone Genette. Hay que recordar que el ordenamiento anacrónico expande el discurso porque exige un mayor número de elementos enunciadores y su efecto es anisocrónico.

En conclusión: si todo esto es correcto, sería válido replantear la clasificación de las unidades funcionales que propone Barthes, y en tal caso, la siguiente podría ser, quizá, una buena proposición:

1) Unidades distribucionales $\left\{\begin{array}{l}\text { nudós narrativos diegéticos (verbos de } \\ \text { acción en los modos de lo réal) }\end{array}\right.$

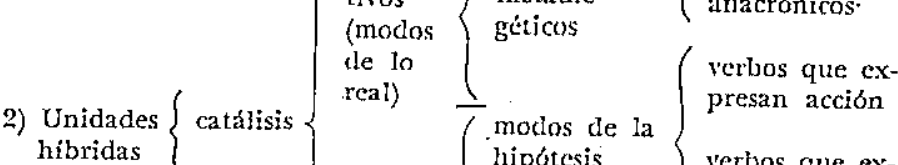

$$
\begin{aligned}
& \text { hibridas } \quad \text { (hipótesis }\left\{\begin{array}{l}
\text { verbos que ex- } \\
\text { pros }
\end{array}\right. \\
& \begin{array}{l}
\begin{array}{l}
\text { nudos } \\
\text { descrip. } \\
\text { tivos }
\end{array}\left\{\begin{array}{l}
\text { hipótesis } \\
\end{array} \quad \begin{array}{l}
\text { verbos que ex- } \\
\text { presan cualidad o } \\
\text { modo de ser }
\end{array}\right.
\end{array} \\
& \begin{array}{l}
\text { 3) Unidades } \\
\text { integradoras }
\end{array}\left\{\begin{array}{l}
\text { indices } \\
\text { informaciones. }
\end{array}\right. \\
& \begin{array}{l}
\text { modos de lo } \\
\text { real }
\end{array}\left\{\begin{array}{l}
\text { verbos que ex: } \\
\text { presan acción }
\end{array}\right.
\end{aligned}
$$


La diferencia entre los nudos narrativos metadiegéticos y los nudos descriptivos con verbos de acción en los modos de lo real, estriba en que estos últimos constituyen expansiones dentro de la misma diégesis, es decir, intradiegéticas; mientras que los primeros forman parte de otra diégesis que altera el ritmo de la narración primaria, pero de otra manera, incidiendo en ella, no desde dentro sino desde fuera.

Helena Beristáin

Seminario de Poútica

Instituto de Investigaciones Filológicas 